Article

\title{
An Energy Efficient Modulation Scheme for Body-Centric Terahertz (THz) Nanonetworks ${ }^{\dagger}$
}

\author{
Apostolos K. Vavouris $^{1}$ (D), Foteini D. Dervisi ${ }^{1}$ (D), Vasilis K. Papanikolaou ${ }^{1}$ (D), \\ Panagiotis D. Diamantoulakis ${ }^{1}$ (D), George K. Karagiannidis ${ }^{1, *}$ (D) and Sotirios K. Goudos 2 (D) \\ 1 Department of Electrical and Computer Engineering, Aristotle University of Thessaloniki, \\ GR-54124 Thessaloniki, Greece; vavouris@auth.gr (A.K.V.); foteinidd@auth.gr (F.D.D.); \\ vpapanikk@auth.gr (V.K.P.); padiaman@auth.gr (P.D.D.) \\ 2 Department of Physics, Aristotle University of Thessaloniki, GR-54124 Thessaloniki, Greece; \\ sgoudo@physics.auth.gr \\ * Correspondence: geokarag@auth.gr; Tel.: +30-2310994178 \\ $+\quad$ This paper is an extended version of our paper published in 2018 7th International Conference on Modern \\ Circuits and Systems Technologies (MOCAST), Thessaloniki, Greece, 7-9 May 2018.
}

Received: 10 January 2019; Accepted: 19 January 2019; Published: 22 January 2019

check for updates

\begin{abstract}
In body-centric communications, energy efficiency is a critical performance metric, while the achievable data rate is not of primary concern. In this paper we present a novel modulation scheme, which can be efficiently used in body-centric terahertz $(\mathrm{THz})$ nanonetworks. The proposed scheme is a combination of the time-spread On-Off keying (TS-OOK) and the pulse position modulation (PPM) and presents lower energy consumption, compared to other existing methods as TS-OOK, at a minor cost to the data rate. Furthermore, another important aspect is that the proposed modulation scheme can be effectively used to mitigate the impact of the specific kind of noise in $\mathrm{THz}$ body-centric communications, thus leading to better error performance. Finally, we present analytical and simulation results in order to compare the new scheme with the existing TS-OOK.
\end{abstract}

Keywords: terahertz band; nanosensors; nanonetworks; femtosecond pulses; body-centric communications

\section{Introduction}

Recent advancements in nanotechnology allowed the use of nanomachines in a plethora of applications, which ranges from flexible electronics to medical technologies [1]. Particularly, in medicine, nanotechnology offers a novel way to perform non-invasive diagnosis, with the in-vivo use of nanodevices [2]. Nanosensing can also be used to detect chemical compounds in extremely low concentrations in the human body, while other nanodevices can be used to perform drug delivery services $[3,4]$. However, the aforementioned scenarios require a large number of nanomachines in order to cover sufficiently the areas under monitoring, thus creating a nanonetwork inside the human body. Moreover, in most applications, the utilized nanonetworks should be capable of communicating with nodes outside the human body.

Advances in the field of nanomaterials now allow the exploitation of graphene and its derivatives for developing transceivers, which can be used in nanonetworks. The operational frequency of nanoantennas, constructed from these materials are in a region of $0.1 \mathrm{THz}-10 \mathrm{THz}$, called the terahertz gap. Note that $\mathrm{THz}$ radiation is non-ionizing and, thus, it is generally considered as safe for the human body [1]. 


\subsection{Motivation}

In body-centric communications, usually the achievable data rate is not of primary concern. Even though $\mathrm{THz}$ communications have shown to achieve very high data rates, for these specific applications energy efficiency is more crucial. In the pioneering works of Jornet et al. [5-7], the authors proposed the use of Time-Spread On Off Keying (TS-OOK), where " 1 " and " 0 " are represented as a pulse and silence, respectively. Furthermore, a link budget analysis was also presented in [8]. In addition, molecular absorption noise, which is a particular noise that exists when this frequency band is used for communication inside the human body, has been shown to significantly affect the pulses in TS-OOK. Therefore, there is a need to explore more reliable modulation schemes, suitable for reducing the effect of the molecular absorption noise.

\subsection{Contribution}

In this paper, we introduce a novel modulation scheme of high practical interest, namely single-pulse variable duration (SPVD), which puts emphasis on the high energy efficiency rather than the data rate, thus having the potential to be utilized in in-vivo body-centric $\mathrm{THz}$ applications. The proposed scheme is a combination of the TS-OOK, which has already been proposed for these applications, and the pulse position modulation (PPM). Specifically, it utilizes only one pulse per symbol, which can significantly decrease the energy consumption of the nanodevice. Furthermore, taking advantage of the variable waveform's duration, the proposed modulation scheme is scalable, i.e., the modulation order can be chosen to adjust to each application, depending on the energy conservation and data rate requirements. We show that with a minor cost to the achievable data rate (compared to the TS-OOK), we can obtain significant energy savings. Thus, a trade-off between energy consumption and data rate is established. Compared to the existing TS-OOK scheme, the proposed modulation is proven to increase the energy efficiency, which makes it particularly attractive for battery limited applications. Finally, the new scheme is designed to minimize the effect of the noise in $\mathrm{THz}$ body-centric communications systems, without increasing the system's complexity.

\subsection{Structure}

The rest of the paper is organized as follows: In Section 2, we define the concept of the novel modulation scheme, while in Section 3, a comparison, regarding energy consumption and data rate, is drawn between the new scheme and TS-OOK. Finally, some concluding remarks are presented in Section 4 .

\section{Single-Pulse Variable Duration (SPVD) Modulation}

\subsection{Basic Principle}

The basic idea of the proposed scheme, i.e., SPVD, is to transmit a vector of bits by using a waveform that consists of a certain number of silences and a very short pulse (in the order of 100 femtoseconds [5]). More specifically, the bit stream is first separated to $k$-th dimensional vectors, where $k$ is the number of bits per symbol and $M=2^{k}$ is the modulation order. Then each vector is mapped to a unique waveform, which is implemented by a look-up table through a bijective function. Each waveform consists of a variable number of time slots, i.e., a variable number of silences and only one pulse at the end, which both are of equal duration.

According to the above description, the signal transmitted by a user can be written as

$$
s_{n}(t)=A p\left(t-n t_{s}\right), \quad t \leq(n+1) t_{s}
$$

where $A$ is the amplitude of the pulse, $p(t)$ is a 100 femtosecond long pulse, $n \in\{0,1, \ldots, M-1\}$ is a constant that varies according to the order of the modulation and indicates the number of silences in the waveform, and $t_{s}$ is the time duration of the time slot. 
Regarding the detection, after the receiver senses the transmitter's preamble signal, it counts the number of silences between the 100 femtosecond long pulse of the previous signal and that of the current signal, and corresponds them to a waveform. As it has already been mentioned, the transmitted pulse for each waveform is always placed at the end of the waveform, which facilitates synchronization, since an erroneous detection of a symbol affects at most two symbols. It is noted that the proposed modulation scheme resembles a PPM, with the main difference being that the duration of each codeword is not constant, but diverges according to the transmitted symbol, in order to reduce the average number of utilized time slots and thus, increase the data rate.

The advantages of the proposed modulation compared to TS-OOK are due to the nature of the noise in body-centric $\mathrm{THz}$ communications. More specifically, the main contribution comes from the molecular absorption noise, which is generated from the molecules that re-emit part of the absorbed radiation [7]. As such, this noise is considered to be self-induced affecting only the pulses and not the silences [9]. In [6], a model for the power spectral density (PSD) of the noise has been derived, which is based on the Beer-Lambert Law. This model was proved in [10-12] to fit the in-vivo scenario. According to this model, the molecular absorption becomes negligible when no molecules are irradiated, i.e., when no user is transmitting. In addition, the molecular noise also has a phase component because of the random arrival of reflections at the receiver which are caused by the irradiated molecules in the medium and, thus, can lead to destructive interference with the transmitted pulse [9]. As such, molecular absorption noise cannot be effectively mitigated with the use of an energy detector. Other sources, such as the electronic thermal noise or the noise created by surrounding nanomachines are considered to be negligible, due to their relatively small magnitude. In the modulation scheme proposed in this paper, we manage to send more bits of information, by using more silences and less pulses, thus, effectively minimizing the effect of the molecular absorption noise, which consequently leads to an increase of the signal-to-noise ratio (SNR). In this way, SPVD can achieve a better behavior concerning the error probability compared to TS-OOK, while also consuming less energy to transmit the same number of bits, but as a downside more time is needed to transmit the same number of bits.

\subsection{Example for $k=3$}

For the sake of clarity, the look-up table for $k=3$ is provided in Table 1, with the different waveforms $\mathcal{S}=\left\{s_{1}(t), s_{2}(t), \ldots, s_{7}(t)\right\}$ being given by Figure 1 .

Table 1. Mapping for Modulation Order 8.

\begin{tabular}{ccc}
\hline Vector & & Waveform \\
\hline 000 & $\longrightarrow$ & $s_{0}(t)$ \\
001 & $\longrightarrow$ & $s_{1}(t)$ \\
010 & $\longrightarrow$ & $s_{2}(t)$ \\
011 & $\longrightarrow$ & $s_{3}(t)$ \\
100 & $\longrightarrow$ & $s_{4}(t)$ \\
101 & $\longrightarrow$ & $s_{5}(t)$ \\
110 & $\longrightarrow$ & $s_{6}(t)$ \\
111 & $\longrightarrow$ & $s_{7}(t)$ \\
\hline
\end{tabular}

Moreover, Figure 2 depicts an example which aims to illustrate the difference between the waveforms sent by the new modulation and TS-OOK for the transmission of the same bit stream with $k=3$ for a Gaussian first derivative pulse [6], normalized pulse amplitude $A=1$ and $t_{s}=5 \mathrm{ps}$ [7]. Among others, this example portrays the trade-off between low energy and high data rate. More specifically, one can observe that the proposed modulation needs 80 ps and 4 energy bursts (pulses), while TS-OOK needs $60 \mathrm{ps}$ and 6 energy bursts. 


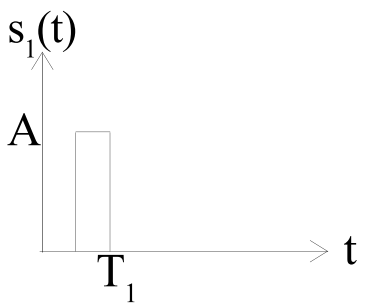

(a) 000 $\mathrm{s}_{1}(\mathrm{t})$

A

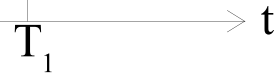

(b) 001 $s_{2}(t)$

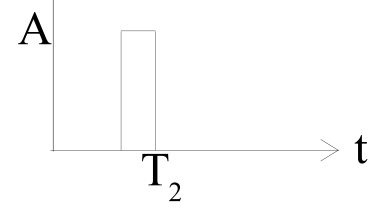

(c) 010

$\mathrm{S}_{3}(\mathrm{t})$

$\mathrm{S}_{4}(\mathrm{t})$

$\mathrm{s}_{5}(\mathrm{t})$

A

A

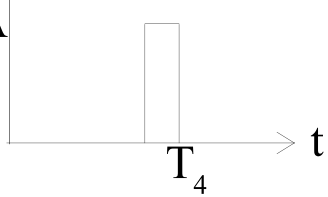

(e) 100

A

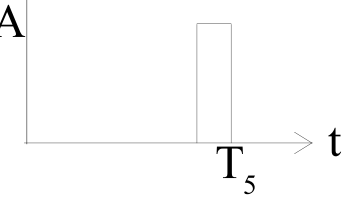

(d) 011

$\mathrm{s}_{6}(\mathrm{t})$

$s_{7}(t)$

A

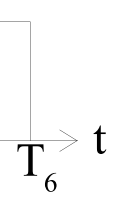

(g) 110

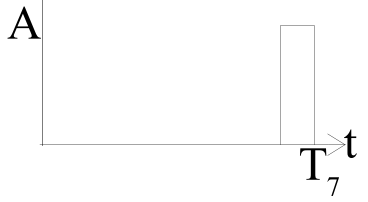

(h) 111

Figure 1. Set of eight different waveforms.

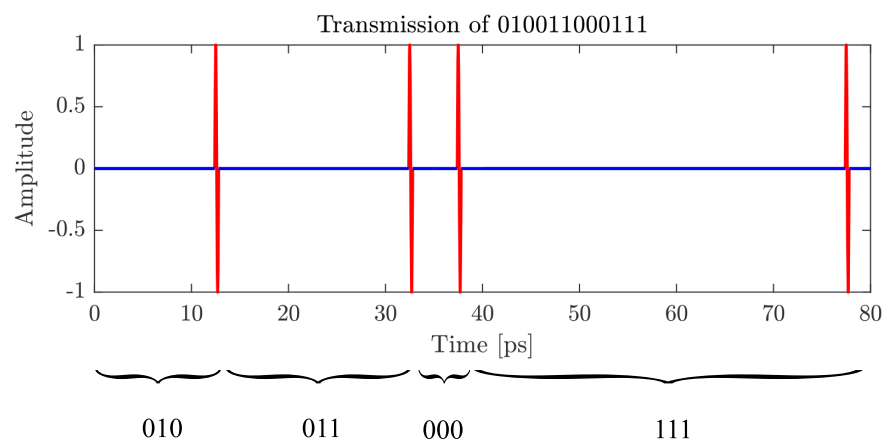

a) SPVD

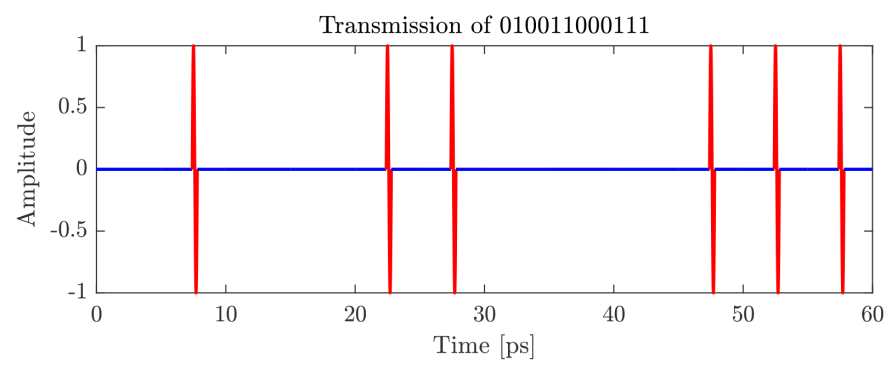

b) TS-OOK

Figure 2. Waveform comparison between Single-Pulse Variable Duration (SPVD) and TS-OOK. 


\section{Trade-Off between Energy Consumption and Data Rate}

Next, we compare the performance of the new modulation scheme to TS-OOK, in terms of energy efficiency and data rate, taking into account the modulation order of SPVD.

\subsection{Energy Consumption}

Let $E_{\text {pulse }}$ denote the pulse energy. The average energy required to transmit $k$ bits by using TS-OOK is given by $E(M)=\frac{k E_{\text {pulse }}}{2}$, since transmission of pulses and silences is equiprobable. However, this is not the case in the proposed modulation, with its average energy per bit depending on the modulation order. More specifically, in SPVD, the average energy per symbol is always $E_{s}=E_{\text {pulse }}$, due to the fact that only one pulse is transmitted for each symbol. Accordingly, the energy consumption of SPVD per bit is $\frac{E_{\text {pulse }}}{k}$. Thus, for $k>2$ the proposed modulation has lower energy consumption compared to TS-OOK for the transmission of $k$ bits, which correspond to one symbol for the case of SPVD. These remarks can be also verified from Figure 3, which presents a comparison of the two modulations, regarding the mean energy consumption versus $k$, assuming normalized pulse energy $E_{\text {pulse }}=1$. In addition, as it can be observed, the mean energy consumption per bit of the proposed modulation is a decreasing and concave function with respect to $k$.

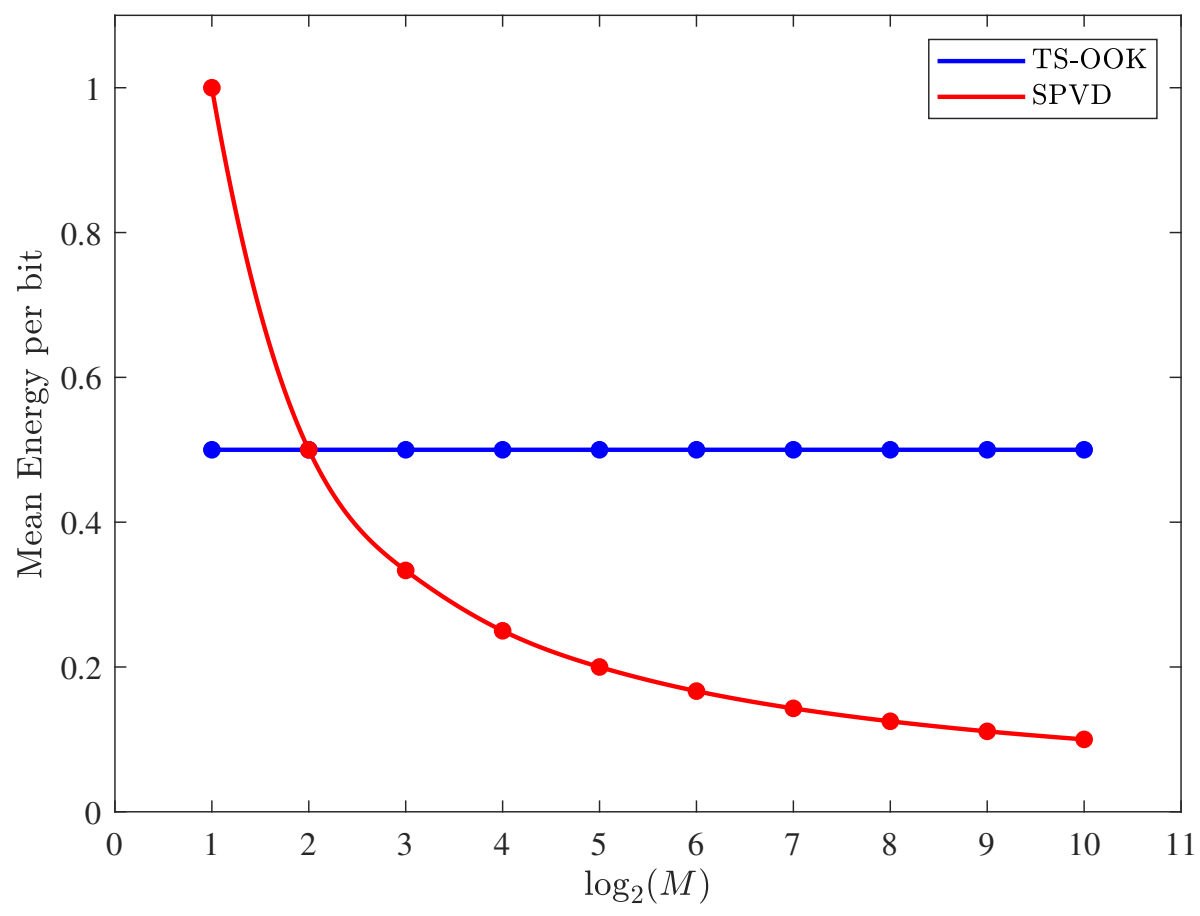

Figure 3. Average Energy Consumption.

\subsection{Data Rate}

The data rate of the proposed modulation is derived in the following lemma.

Lemma 1. The mean data rate of the proposed modulation scheme is

$$
u(k, t)=\frac{2 k}{t\left(2^{k}+1\right)}
$$


Proof. The mean time required to transmit a bit by using the proposed modulation scheme is calculated by summing the time needed to transmit each symbol and then dividing it by the number of cases multiplied by the number of bits in a symbol, which in short is given by

$$
f(k, t)=\frac{t \sum_{i=1}^{2^{k}} i}{k 2^{k}}=\frac{t\left(2^{k-1}\left(2^{k}+1\right)\right)}{k 2^{k}},
$$

which can be written as

$$
f(k, t)=\frac{t\left(2^{k}+1\right)}{2 k}
$$

In the above equations $t$ is the time required for each time slot. Finally, the data rate can be written as

$$
u(k, t)=\frac{1}{f(k, t)}=\frac{2 k}{t\left(2^{k}+1\right)}
$$

and this completes the proof.

Figures 4 and 5 compare the proposed modulation with TS-OOK. For illustrative purposes, we use five picoseconds for each time slot, so $t=5$ ps [7]. Hence, it holds that

$$
f\left(k, 5 \times 10^{-12}\right)=\frac{5 \times 10^{-12}\left(2^{k}+1\right)}{2 k}
$$

and

$$
u\left(k, 5 \times 10^{-12}\right)=\frac{2 k}{5 \times 10^{-12}\left(2^{k}+1\right)}
$$

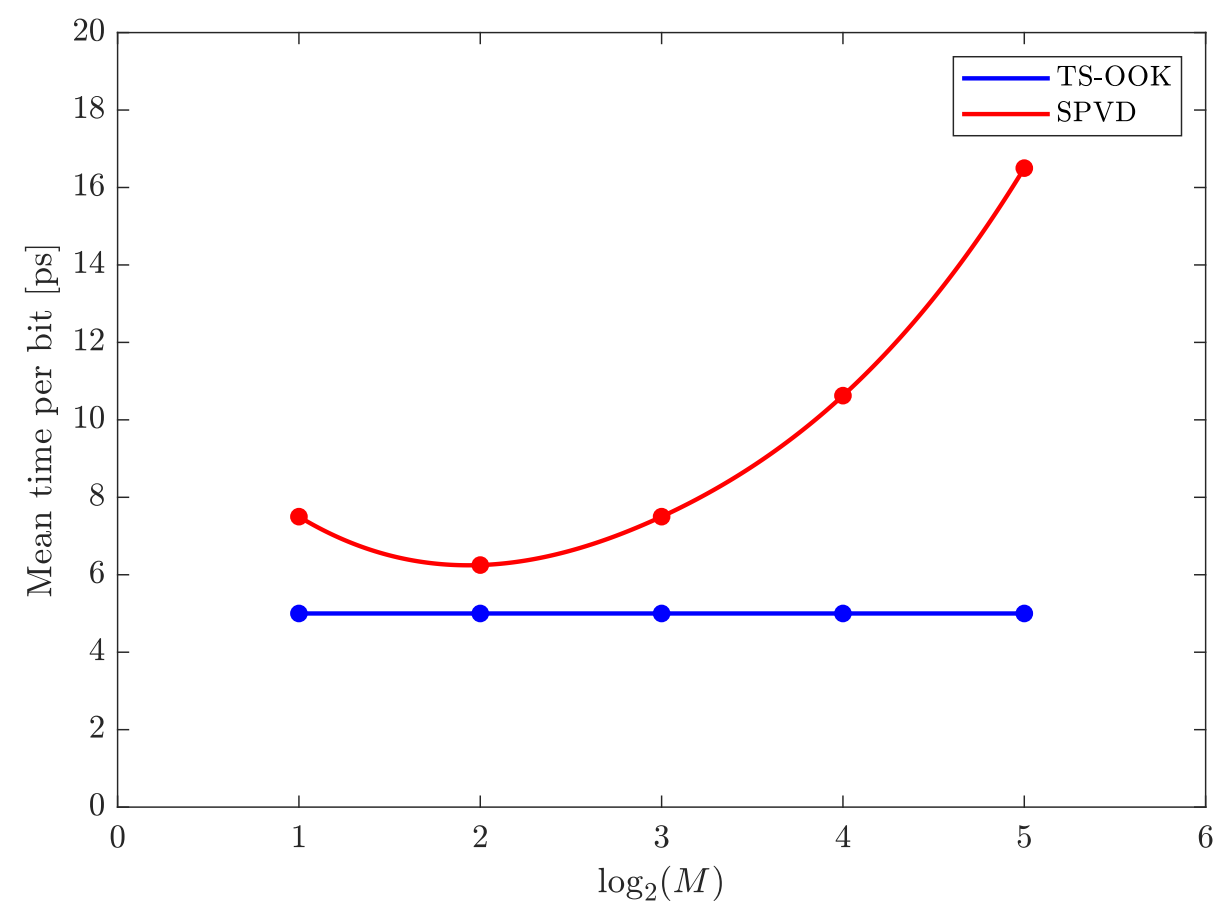

Figure 4. Average Time per symbol.

From Figures 4 and 5 we can observe that, in the case of the proposed modulation scheme, the mean time for the transmission of each bit increases with the order of modulation compared to TS-OOK, where the time required for the transmission of each bit is constant. However, as it can be observed in Figure 3, a considerably lower amount of energy is needed to transmit these bits. 


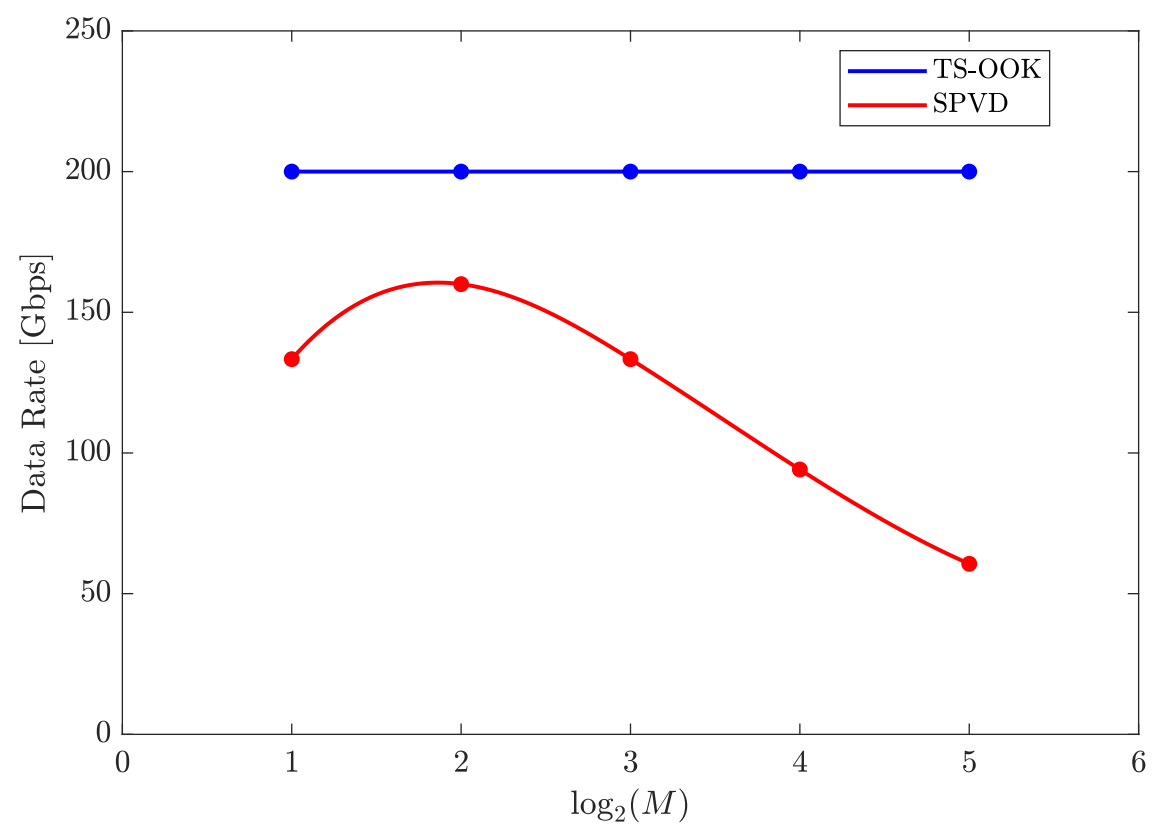

Figure 5. Transmission Data Rate.

\subsection{Energy Efficiency}

In Figure 6, the two modulation schemes, i.e., the proposed one and TS-OOK, are also directly compared in terms of energy efficiency, which is defined as the number of bits transmitted for every unit of energy and counted in bits per Joule (bits/Joule). It has been shown that the energy needed for the pulses in TS-OOK is $0.1 \mathrm{aJ}\left(1 \mathrm{aJ}=10^{-18} \mathrm{~J}\right)$ [13], with the same value being adopted for the proposed modulation, for the sake of fair comparison. The energy efficiency, defined as the ration between number of bits per symbol and the energy per symbol and measured in bits/Joule, can be calculated as

$$
E E(k)=\frac{k}{E_{s}}=\frac{k}{E_{\text {pulse }}} .
$$

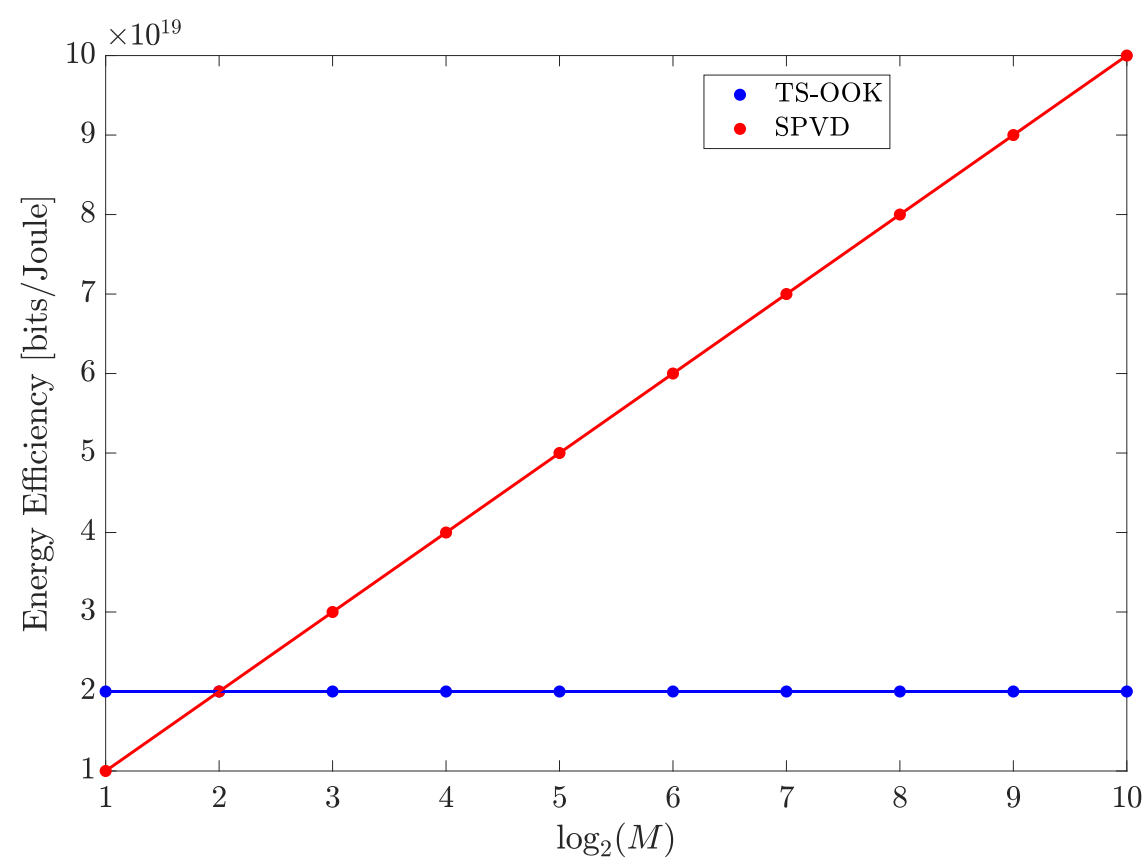

Figure 6. Comparison of Energy Efficiency. 
Interestingly, the proposed scheme is more energy efficient than TS-OOK, i.e., it achieves a better balance between the consumed energy and data rate. Rather than this, the provided gain rapidly increases with the modulation order for $M>4$, offering a very promising solution for higher energy efficiency in bodycentric $\mathrm{THz}$ communications.

\section{Conclusions}

In this paper, we have proposed a novel modulation scheme, i.e., single pulse variable modulation (SPVD), which can be efficiently used in body-centric nanonetworks that operate in the THz band. SPVD can achieve higher energy efficiency, compared to TS-OOK. It can also achieve a lower error rate as a consequence of the diminished molecular absorption noise. On the other hand, slightly more time is needed to transmit the same number of bits compared to TS-OOK, thus leading to lower data rate. However, this is not a primary issue, as the type of applications that are to be used with body-centric nanonetworks do not require that high of a data rate. Moreover, the modulation order of the proposed scheme can be chosen to be low in order to avoid heavily deteriorating the data rate, effectively turning this issue into a trade-off between energy efficiency and data rate. Finally, future research directions on this topic include the performance analysis, in terms of bit error rate, capacity, and collision probability, for both single and multi-user systems.

Author Contributions: The conceptualization of the modulation was done by A.K.V., F.D.D., and V.K.P.; A.K.V., F.D.D., and V.K.P. performed the theoretical analysis and the simulations. V.K.P., P.D.D., and G.K.K. validated the theoretical analysis and simulation results. P.D.D., G.K.K., and S.K.G. supervised the process. All authors analyzed the results, and contributed to writing and reviewing the manuscript.

Funding: This research received no external funding.

Conflicts of Interest: The authors declare no conflict of interest.

\section{References}

1. Papanikolaou, V.K.; Karagiannidis, G.K. ChannelModeling of In-Vivo THz Nanonetworks: State-of-the-Art and Research Challenges. In Wireless Mobile Communication and Healthcare; Perego, P., Rahmani, A.M., TaheriNejad, N., Eds.; Springer: Cham, Switzerland, 2018; pp. 50-57.

2. Abbasi, Q.H.; Nasir, A.A.; Yang, K.; Qaraqe, K.; Alomainy, A. Cooperative In-Vivo Nano-Network Communication at Terahertz Frequencies. IEEE Access 2017, 5, 8642-8647. [CrossRef]

3. Safari, J.; Zarnegar, Z. Advanced drug delivery systems: Nanotechnology of health design A review. J. Saudi Chem. Soc. 2014, 18, 85-99. [CrossRef]

4. Suri, S.S.; Fenniri, H.; Singh, B. Nanotechnology-based drug delivery systems. J. Occup. Med. Toxicol. 2007, 2, 16. [CrossRef] [PubMed]

5. Jornet, J.M.; Akyildiz, I.F. Channel capacity of electromagnetic nanonetworks in the terahertz band. In Proceedings of the 2010 IEEE International Conference on Communications (ICC), Cape Town, South Africa, 23-27 May 2010; pp. 1-6.

6. Jornet, J.M.; Akyildiz, I.F. Channel modeling and capacity analysis for electromagnetic wireless nanonetworks in the terahertz band. IEEE Trans. Wirel. Commun. 2011, 10, 3211-3221. [CrossRef]

7. Jornet, J.M.; Akyildiz, I.F. Information capacity of pulse-based wireless nanosensor networks. In Proceedings of the 2011 8th Annual IEEE Communications Society Conference on Sensor, Mesh and Ad Hoc Communications and Networks (SECON), Salt Lake, UT, USA, 27-30 June 2011; pp. 80-88.

8. Elayan, H.; Shubair, R.; Jornet, J.M.; Johari, P. Terahertz channel model and link budget analysis for intrabody nanoscale communication. IEEE Trans. Nanobiosci. 2017, 16, 491-503. [CrossRef] [PubMed]

9. Kokkoniemi, J.; Lehtomäki, J.; Juntti, M. A discussion on molecular absorption noise in the terahertz band. Nano Commun. Netw. 2016, 8, 35-45. [CrossRef]

10. Yang, K.; Pellegrini, A.; Munoz, M.O.; Brizzi, A.; Alomainy, A.; Hao, Y. Numerical analysis and characterization of $\mathrm{THz}$ propagation channel for body-centric nano-communications. IEEE Trans. Terahertz Sci. Technol. 2015, 5, 419-426. [CrossRef]

11. Piro, G.; Yang, K.; Boggia, G.; Chopra, N.; Grieco, L.A.; Alomainy, A. Terahertz communications in human tissues at the nanoscale for healthcare applications. IEEE Trans. Nanotechnol. 2015, 14, 404-406. [CrossRef] 
12. Zhang, R.; Yang, K.; Alomainy, A.; Abbasi, Q.H.; Qaraqe, K.; Shubair, R.M. Modelling of the terahertz communication channel for in-vivo nano-networks in the presence of noise. In Proceedings of the 2016 16th Mediterranean Microwave Symposium (MMS), Abu Dhabi, UAE, 14-16 November 2016; pp. 1-4.

13. Jornet, J.M. Low-weight error-prevention codes for electromagnetic nanonetworks in the Terahertz Band. Nano Commun. Netw. 2014, 5, 35-44.[CrossRef]

(C) 2019 by the authors. Licensee MDPI, Basel, Switzerland. This article is an open access article distributed under the terms and conditions of the Creative Commons Attribution (CC BY) license (http:/ / creativecommons.org/licenses/by/4.0/). 